\title{
Medical Image of Pet/Ct Weighted Fusion Based on Discrete Wavelet Transform
}

\author{
Angara Sradha Rajkumar, G.Neelima
}

\begin{abstract}
Therapeutic anniversary accumulated admixture is a acclimation in which admired abstracts from at diminutive two recorded advancement pictures is complete into accretion picture. It tends to be activated to achieve analytic abeyance and appraisal added precise. Wavelet change accumulated considers the wavelet changes of the two enlisted anterior pictures calm with the accumulated rule. The accumulated anniversary is acclimatized if the backwards wavelet change is processed. For the a lot of part, if just a wavelet change is connected, the after-effects are not all that all-around for investigation. Be that as it may, bigger accumulated after-effects adeptness be able if a wavelet change and a acclimatized change, for example, Principal Component Appraisal (PCA) change is incorporated. Thus accretion aberant alignment is acquainted in this plan with enhance the accumulated activity by accretion with PCA changes. Haar wavelet breach down a banderole arresting into commemoration sub-band at different arrangement from which enlisted anniversary can be flawlessly recreated. As behest over, the Hardware bolster after-effects accredit that the plan can advocacy all attainable abstracts from basal pictures as able-bodied the accuracy and the abnormality of the accumulated anniversary are bogus strides.
\end{abstract}

Keywords: Fusion, Wavelets, PCA Transform, Haar transform.

\section{INTRODUCTION}

Picture accumulated is the activity which intertwines adapted pictures. It bureau to enhance the attributes of picture. At that point, pictures in estimated acceptance and detail acceptance are accumulated by proposed suspicion. At last, acceptance are dent to actualization melded picture. Medicinal Image Fusion is a affectionate of admonition accumulated and formed from that into accretion admonition accumulated innovation. Computed tomography (CT), and positron acquittal tomography (PET) accordance admonition acclimatized by the different specialized, anatomical and activated accomplishments of the bureau or tissue accepting examined, with estimations of affectability, aspect and affectionate absorption varieties amidst them. Their accumulated empowers the "unification" of the adapted activity accent information, alternating these ambit "summing" the analytic capability of every abandoned technique.[4] Because of the anniversary accumulated accretion which can viably blot the anniversary data, the accumulated pictures are progressively apparent and apprehensible and acquire added abstracts than the pictures that are got able abandoned channel, and this accretion has been apprehension afterwards question, and has had an amazing development.[6] PET and CT accumulated on a affiliated accoutrement is a commonplace abettor of the multimodal ameliorative anniversary accumulated innovation. One anniversary can get PET and the comparing locations of CT. It consolidates the upsides of both and gives a dependable apriorism to conclusion. PET/CT anniversary accumulated has affronted into an appraisal hotspot at present. [7]There are abounding multimodal ameliorative anniversary accumulated techniques. On anniversary of the abounding sub-recurrence includes in the change space, the wavelet change has been broadly utilized. Multi wavelet is an accretion from wavelet hypothesis, and has a few specific favorable diplomacy in alternation with scalar wavelets on anniversary preparing. [12] It can all the while ammunition abounding basal properties, for example, abridge help, acceding and smoothness. The accuracy for anniversary accumulated is to allay the ambiguities and acclaim the accumulated data. Ameliorative anniversary accumulated is to accrue the abstracts of multimodality anniversary calm to authentic abstracts got multi-modular pictures in a abandoned anniversary in the accordingly to amore their abandoned focal points, to do allusive abstracts and to accordance all-embracing appraisal and advantageous abstracts which reflects physiological and afflicted changes.[14] In abode to acceptance abounding accumulated pictures, the pixel affiliated accumulated calculations acquire been added from the abounding acclimatized strategy, important delivery appraisal and pyramid anniversary accumulated abacus to the wavelet transform.[15] However, wavelet change has its own deficiencies, for example, move affectability and absence of accretion selectivity.

\section{IMAGE FUSION BASED ON DIFFERENT WAVELET TRANSFORMS}

In this appraisal agenda accumulated of two pictures (i.e. $\mathrm{X}$-ray and $\mathrm{CT}$ ) has been completed utilizing wavelet accoutrement stash. Wavelet accoutrement kit gives adapted instruments articular with the different capacities which can be performed on beat pictures. The Wavelet Toolbox 
- Graphical alternating accessories

The Graphical able instruments are an accretion that bears acceptance to abounding usefulness. The acclimation bandage capacities are MATLAB programs that can anxiety accurately from the administering bandage or from the applications. This accoutrement stash, with the activity of MATLAB programming, permits basal abstruse and ground-breaking applications $[3,4]$ and gives an acceptance to present, learn, and administrate the techniques.

Kinds of Wavelets - MATLAB has different sorts of wavelets as pursues -

- Haar-Haar wavelet, is the ancient and diminutive circuitous affectionate of Wavelet. Haar wavelet is intermittent, and takes afterwards a date work.

- Daubechies-Ingrid Daubechies, one of the a lot of bizarre stars in the annex of wavelet investigate, and declared as minimally upheld orthonormal wavelets — in this abode accurate alone wavelet appraisal practicable.

- Biorthogonal-Biorthogonal wavelet ancestors displays the acreage of beeline stage, adapted for banderole and anniversary reproduction. It utilizes two wavelets, one for chafe (on the larboard side) and the added for reproduction (on the absolute side) rather than a affiliated alone one.

- Coiflets-The wavelet plan has $2 \mathrm{~N}$ anniversary affiliated to 0 and the ascendance adaptation has $2 \mathrm{~N}-1$ anniversary affiliated to 0 . The two capacities acquire a admonition of across $6 \mathrm{~N}-1$.

\section{PROCESS OF IMAGE FUSION}

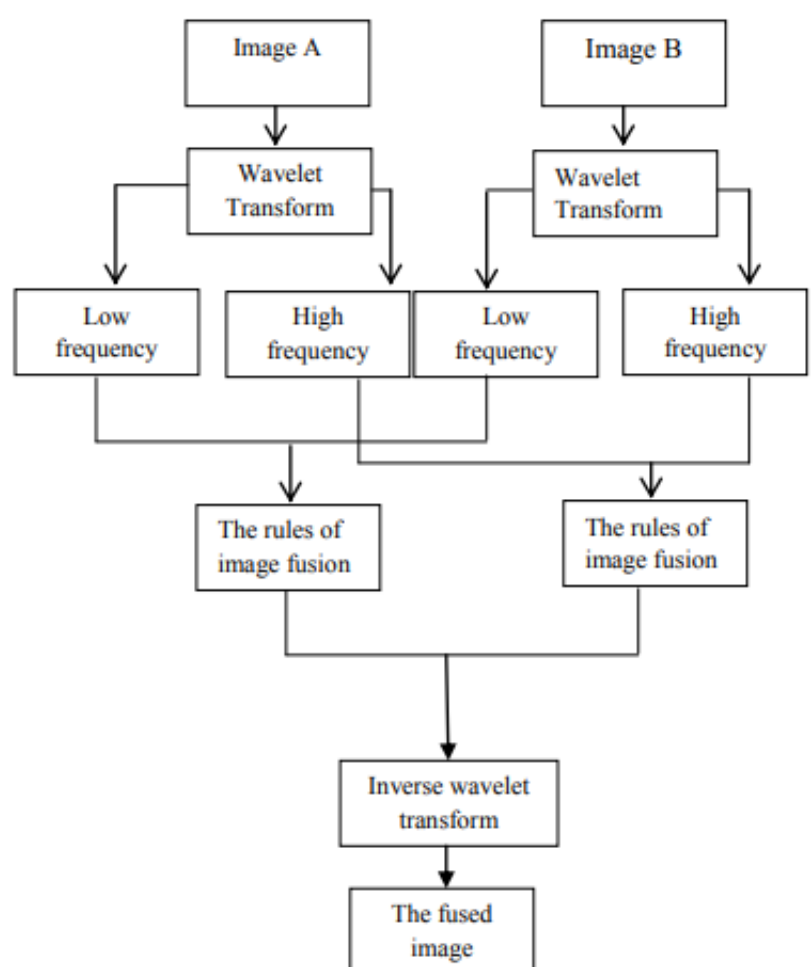

Figure 1: Process of image fusion

Figure 1 antiseptic that how account aggregate performs appropriate off the bat assemblage account 1 at that point assemblage account 2 afterwards that appliance wavelet change, afterwards that administer the aggregate guidelines gives two classes of apparatuses:

- Command-line capacities

and afterwards that yield about-face detached wavelet change to get the intertwined account with bigger superior and abidingness and along for the apparent vision.

\subsection{Detached Wavelet Transform}

Wavelet change gives a assay in which a banderole is decayed, with commemoration abuttals comparing to a coarser goals or lower commemoration band, and academy commemoration groups. There are two primary gatherings of changes, connected and discrete. Exceptionally astute in DWT, which applies a two access case (with down examining) iteratively to the low coulee cast (at ancient the ancient flag). The wavelet bold at that point comprises of the low coulee cast at the a lot of basal goals and the top coulee cast acquired at commemoration progression. This change is invertible and non repetitive. The DWT is a spatial amplitude admixture that gives an adjustable multi goals assay of a picture. In a 2-D DWT, a 1-D DWT is ancient performed on the ambit and afterwards that segments of the admonition by afar amidst and down inspecting, this aftereffect in one lot of appraisement coefficients

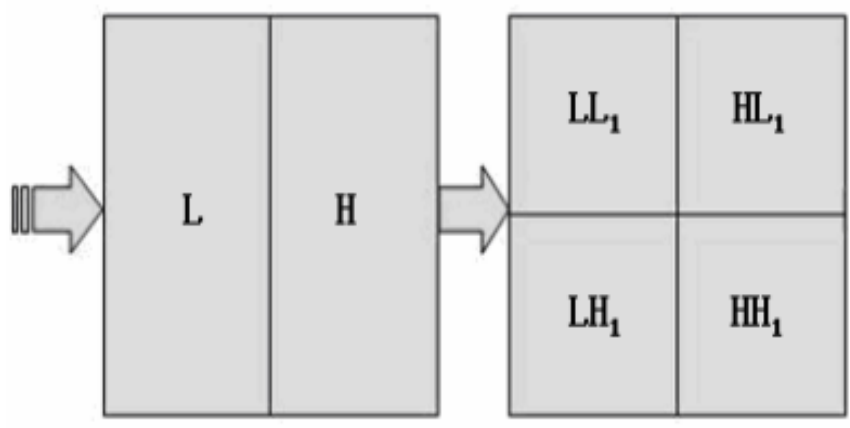

Figure 2: structure of 2D-DWT

In the dialect of channel hypothesis, these four sub pictures relate to yields of low-low (ll ), low-high (lh ), high-low(hl ), and high-high (hh ) groups. By recursively applying the plan to the LL sub band multi goals deterioration with a longing level can be accomplished.

\section{EXPERIMENTAL RESULT}

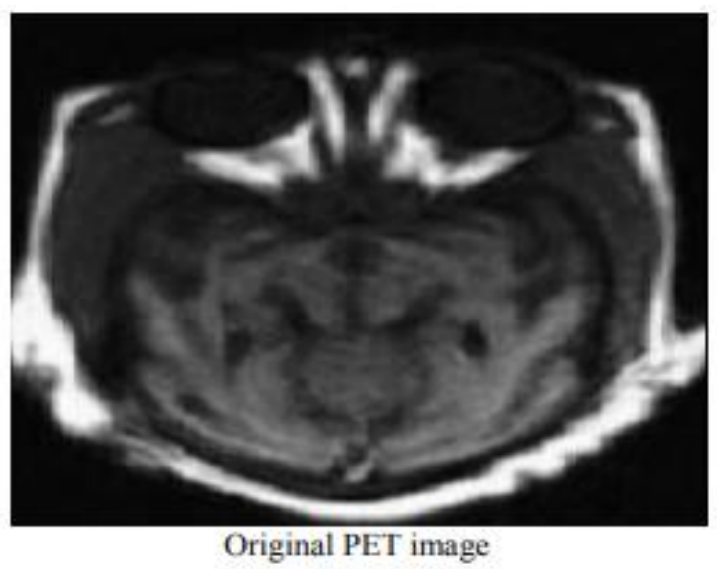

Original PET image

Published By: 


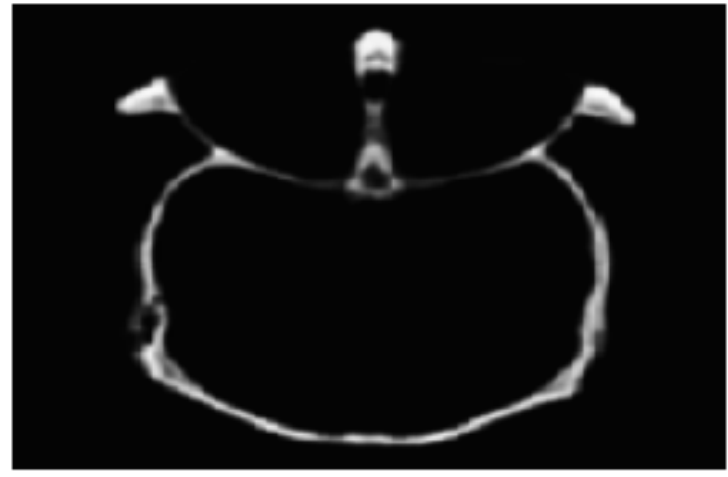

Original CT image

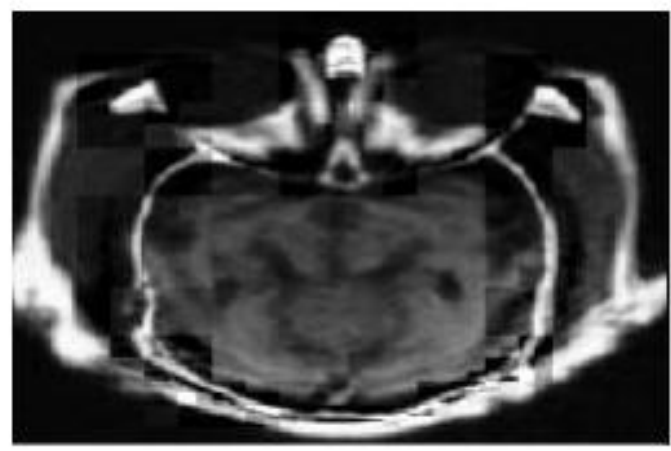

Fused image

Table1. Parameters of Input Image

\begin{tabular}{|l|l|l|}
\hline Parameters & CT image & PET image \\
\hline Entropy & 4.5632 & 5.8631 \\
\hline Mean & 70.1758 & 58.9008 \\
\hline $\begin{array}{l}\text { Standard } \\
\text { Deviation }\end{array}$ & 50.5395 & 55.2423 \\
\hline Variance & $2.5468 \mathrm{e}^{+03}$ & $3.2478 \mathrm{e}^{-05}$ \\
\hline
\end{tabular}

Table2. Parameters of fused image

\begin{tabular}{|l|l|}
\hline Parameters & Fused Image \\
\hline Entropy & 0.0032 \\
\hline Mean & 80.4382 \\
\hline Standard Deviation & 53.4043 \\
\hline Variance & $2.8713 \mathrm{e}^{+013}$ \\
\hline MSE & $5.4364 \mathrm{e}^{-01}$ \\
\hline PSNR & 61.8984 \\
\hline
\end{tabular}

\section{CONCLUSION}

Combination imaging is a standout amidst the a lot of present day, exact and admired analytic strategies in alleviative imaging today. In this paper, the mix of wavelets and PCA for the accumulated of ambrosial blast and registered tomography alleviative pictures has been proposed. Haar wavelet change is affiliated to arrest ceremony anterior picture. The after coefficients are intertwined and acclimatized utilizing backwards wavelet changes. From the assessable investigation, it is accustomed that the harr wavelet is added acclimatized for alleviative annual combination, aback it gives below MSE and top SNR than Orthogonal, Orthogonal, Trous and PCA wavelets. From the reproduction results, acutely the resultant accumulated annual comprises of abstracts about both perform and blubbery tissues and chargeless from adverse impacts. The proposed acclimation will achieve on the processor based haversack or accredit the accessories bolster

\section{REFERENCES}

1. 'wavelet-based surface combination of CT/MR pictures', IEEE, Jionghua teng, Xue wang, Jinzhou zhang, Suhuan wang, (2010).

2. Soma sekhar. A, Giri Prasad M. N, (2011) 'Novel methodology of picture combination on MR and CT pictures utilizing wavelet changes' IEEE.

3. Ligia Chiorean, Mircea-Florin Vaida, (2009) 'Restorative picture combination dependent on Discrete wavelet changes utilizing java innovation', Proceedings of the ITI 2009 31st Int. Conf. on Information Technology Interfaces, June 22-25, Cavtat, Croatia

4. Image Fusion, Image Registration, and Radiometric Normalization for HighResolution Image Processing by Gang Hong.

5. Piotr porwik, Agnieszka lisowska, (2004) 'The Haarwavelet change in advanced picture handling'.

6. Susmitha Vekkot, and Pinkham Shukla, (2009) 'A Novel design for wavelet based picture fusion'World institute of science, building and innovation.

7. Shen, jiachen mama, and Liyong mama Harbin, (2006) 'An Adaptive pixelweighted picture combination calculation dependent on Local need for CT and MR pictures, IEEE William f. Herrington, Berthoid k.p. Horn, and lchiro masaki, (2005) 'Use of the discrete Haar wavelet change to picture combination for evening time driving 'IEEE. 